\title{
Maximum-entropy ansatz for nonlinear-response theory
}

\author{
Jeppe C. Dyre* \\ Noyes Laboratory, University of Illinois at Urbana-Champaign, Urbana, Illinois 61801
}

(Received 13 February 1989)

\begin{abstract}
An approximate nonlinear-response theory is derived by maximizing the information theoretic entropy in the space of fluctuations $J(t)$ of the degree of freedom of interest, subject to constantenergy dissipation in the external field. The resulting formalism expresses all cumulant averages of $J(t)$ in an external field in terms of equilibrium cumulants.
\end{abstract}

Linear-response theory ${ }^{1-7}$ is fundamental to much present-day work in solid-state physics, chemical physics, plasma physics, etc. Its usefulness rests in the fact that the response to an external field to first order is determined by an equilibrium autocorrelation function. The extension of linear-response theory to include higherorder terms had been discussed already in the 1950 s. $^{6,8}$ In contrast to the linear case, the higher-order terms cannot be expressed in terms of equilibrium correlation functions of the degree of freedom under consideration. ${ }^{9}$ The exact nonlinear-response theory is complex and has found little application. There is also the question of the convergence of the formal expansion in the external field. Strong fields may induce, e.g., phase transitions or selforganization, information about which one does not expect to be hidden in the equilibrium fluctuations. Despite these reservations, one may ask whether a theory that predicts the nonlinear response from equilibrium fluctuations does exist, at least as a reasonable approximation applicable not too far from equilibrium. In an attempt to answer this question, this paper discusses an ansatz for nonlinear-response theory which generalizes a littleknown theory due to Stratonovich. ${ }^{10}$ The ansatz is derived from the maximum-entropy principle. Some simple examples are given and the validity of the ansatz is discussed. Finally, it is argued that the ansatz represents the simplest way a system may become nonlinear.

The degree of freedom of interest is denoted by $Q$; it may be microscopic or macroscopic. The underlying dynamics is assumed to be classical and $Q$ is assumed to be invariant under time reversal. $Q$ couples to an external field $E$ via a term $-E Q$ in the Hamiltonian. Only the case of a time-independent external field will be considered. It is convenient to discuss response and fluctuations in terms of $J(t) \equiv \dot{Q}(t)$. In general, one is interested in not only the average $\langle J\rangle_{E}$ but also in the autocorrelation function and perhaps higher-order averages of $J(t)$ in the external field. To calculate these quantities one needs to know the probability of any $J(t)$ fluctuation in the external field $P_{E}[J(t)]$. As mentioned above, even a complete knowledge of the equilibrium fluctuations $P_{0}[J(t)]$ in general is not enough to determine $P_{E}[J(t)]$. The task of finding $P_{E}[J(t)]$ may be viewed as a missinginformation problem. In an external field there is a certain rate of energy dissipation. Suppose no more than this and $P_{0}[J(t)]$ are known. The maximum-entropy formalism is a general method that, based on the insufficient information available, gives a least-biased estimate of probabilities. ${ }^{11,12}$ In applications to a continuous problem a reference measure is needed. In the present case, in order to have a smooth transition to the zero-field case this measure must be $P_{0}[J(t)]$. The maximum-entropy requirement leads to ${ }^{11,12}$

$$
P_{E}[J(t)]=N^{-1} P_{0}[J(t)] \exp \left(-\lambda E \int_{-\infty}^{\infty} J(t) d t\right),
$$

where $N$ is a normalization constant and $\lambda=\lambda(E)$ is a Lagrangian multiplier. Normally, one would now proceed to determine $\lambda$ from the constraint, i.e., the energy dissipation rate. In this case, however, $\lambda$ is determined by fundamental requirements of statistical mechanics. As shown by Bochkov and Kuzovlev, ${ }^{13,14}$ the time-reversal invariance of the microscopic equations of motion implies

$$
\frac{P_{E}[J(t)]}{P_{E}[-J(-t)]}=\exp \left[\beta E \int_{-\infty}^{\infty} J(t) d t\right] \text {, }
$$

where $\beta$ is the inverse temperature. Equation (2) is derived by assuming thermal equilibrium at $t=-\infty$ followed by a decoupling of the heat bath and evolution according to the canonical equations of motion for the system interacting with the external field. This assumption is identical to that used by Kubo in deriving linearresponse theory. ${ }^{6}$ Equation (2) is also valid for any system that is described by a master equation; in this case, Eq. (2) expresses the principle of detailed balance. Since $P_{0}[J(t)]$ is time-reversal invariant, Eqs. (1) and (2) imply $\lambda=-\beta / 2$ for all $E$, i.e.,

$$
P_{E}[J(t)]=N^{-1} P_{0}[J(t)] \exp \left[\frac{\beta}{2} E \int_{-\infty}^{\infty} J(t) d t\right]
$$

Equation (3) allows one to calculate all higher-order cumulant averages of $J$ in the external field, in terms of equilibrium cumulants. The cumulants are defined by the generating functional 


$$
\begin{aligned}
D[u(t) ; E]= & \ln \left\langle\exp \left[\int_{-\infty}^{\infty} u(t) J(t) d \tau\right)\right\rangle_{E} \\
= & \sum_{n=1}^{\infty} \frac{1}{n !} \int_{-\infty}^{\infty} d t_{1} \cdots d t_{n}\left\langle J\left(t_{1}\right), \ldots, J\left(t_{n}\right)\right\rangle_{E} \\
& \times u\left(t_{1}\right) \cdots u\left(t_{n}\right)
\end{aligned}
$$

and Eq. (3) implies

$$
D[u(t) ; E]=D[u(t)+(\beta / 2) E ; 0]-D[(\beta / 2) E ; 0] .
$$

From Eq. (5) the nonequilibrium cumulants may be found as derivatives with respect to $u(t)$ at $u \equiv 0$.

For the average of $J$ one finds from Eq. (5)

$$
\begin{aligned}
&\langle J\rangle_{E}=\sum_{n=0}^{\infty} \frac{1}{n !}\left[\frac{\beta E}{2}\right]^{n} \int_{-\infty}^{\infty} d t_{1} \cdots d t_{n} \\
& \times\left\langle J(0), J\left(t_{1}\right), \ldots, J\left(t_{n}\right)\right\rangle_{0} .
\end{aligned}
$$

The first-order term is that predicted by linear-response theory. Note that, for a macroscopic $Q$, Eq. (6) is consistent also in the thermodynamic limit since cumulants are additive for independently fluctuating quantities. An identity for the $(n+1)$ th cumulant average of $\Delta Q(t)=Q(t)-Q(0)$,

$$
\begin{aligned}
\lim _{t \rightarrow \infty} t^{-1}\left\langle\Delta Q^{(n+1)}(t)\right\rangle_{0} & \\
& =\int_{-\infty}^{\infty} d t_{1} \cdots d t_{n}\left\langle J(0), J\left(t_{1}\right), \ldots, J\left(t_{n}\right)\right\rangle_{0},
\end{aligned}
$$

allows one to rewrite Eq. (6) as

$$
\langle J\rangle_{E}=\left.\lim _{t \rightarrow \infty} t^{-1} \frac{d}{d u} \ln \left\langle e^{u \Delta Q(t)}\right\rangle_{0}\right|_{u=(\beta / 2) E} .
$$

As a simple example of Eq. (7), consider a particle performing a random walk in one dimension on a lattice with lattice constant $a$. $Q$ is the coordinate and $J$ the ve- locity of the particle. If there are $N$ jumps in time $t$, it is easy to see that $\left\langle e^{u \Delta Q(t)}\right\rangle_{0}=\cosh ^{N}(u a)$. Since $N$ is Poisson distributed around a mean $\bar{N}=\Gamma t$, one has

$$
\begin{aligned}
\left\langle e^{u \Delta Q(t)}\right\rangle_{0} & =e^{-\bar{N}} \sum_{N=0}^{\infty} \frac{\bar{N}^{N}}{N !} \cosh ^{N}(u a) \\
& =\exp \{\bar{N}[\cosh (u a)-1]\},
\end{aligned}
$$

which implies

$$
\langle v\rangle_{E}=\Gamma a \sinh [(\beta / 2) E a] .
$$

This is the correct result if the random walk is realized as the low-temperature limit of a particle in a symmetric periodic potential obeying a Langevin equation; the wellknown Eq. (9) follows from the fact that the field lowers the barrier for jumps in the direction of $E$ and increases the barrier for jumps opposite $E$. As another example of Eq. (7), consider the case when $Q$ is the $x$ coordinate of an atom in a liquid. Then Eq. (7) expresses the velocity of the atom in a field in the $x$ direction acting only on the atom, in terms of the equilibrium incoherent intermediate scattering function $F_{s}(k, t)$ :

$$
\langle v\rangle_{E}=\left.\lim _{t \rightarrow \infty}(i t)^{-1} \frac{\partial}{\partial k} \ln F_{s}(k, t)\right|_{k=\beta E / 2 i} .
$$

For simple Gaussian diffusion, $F_{s}(k, t)=\exp \left(-D k^{2} t\right) .^{15}$ In this case, the response is exactly linear and Eq. (10) reduces to the Nernst-Einstein relation between mobility and diffusion constant. A more realistic fit to experiment is the prediction of the jump-diffusion model ${ }^{15,16}$

$$
F_{s}(k, t)=\exp \left[-D k^{2} t /\left(1+D k^{2} \tau_{0}\right)\right] .
$$

From this, Eq. (10) predicts the onset of nonlinearity at fields for which $E l \beta \simeq 1$, where the length $l=\sqrt{D \tau_{0}}$ in fits to data is of order-1 interatomic spacing.

For the external field autocorrelation function, Eq. (5) implies

$$
\begin{aligned}
\langle J(0), J(t)\rangle_{E}= & \langle J(0), J(t)\rangle_{0}+\frac{\beta E}{2} \int_{-\infty}^{\infty} d t_{1}\left\langle J(0), J(t), J\left(t_{1}\right)\right\rangle_{0} \\
& +\frac{1}{2}\left[\frac{\beta E}{2}\right]^{2} \int_{-\infty}^{\infty} d t_{1} d t_{2}\left\langle J(0), J(t), J\left(t_{1}\right), J\left(t_{2}\right)\right\rangle_{0}+\cdots
\end{aligned}
$$

An example is the case when $J$ is the electric current. For a system with frequency-independent conductivity the zeroth-order term is a $\delta$ function, giving rise to white noise. In most cases the next term is zero by symmetry and the so-called excess current noise is given by the second-order term. Excess current noise is ubiquitous and usually has a $1 / f$ spectrum..$^{17-19} \mathrm{~A}$ number of random-walk models have been studied to gain a better understanding of the phenomenon. In these models one finds ${ }^{20-23}$

$$
\begin{aligned}
& \langle J(0), J(t)\rangle_{E} \\
& =(\beta E)^{2} \int_{0}^{t} d t_{2} \int_{0}^{t_{2}} d t_{1}\left\langle J(0), J(t), J\left(t_{1}\right), J\left(t_{2}\right)\right\rangle_{0}+\cdots \\
& (t>0),
\end{aligned}
$$

which is equivalent to Eq. (11) because in random-walk models $\left\langle J\left(t_{1}\right), J\left(t_{2}\right), J\left(t_{3}\right), J\left(t_{4}\right)\right\rangle$ is nonzero only if $t_{1} \approx t_{2}$ and $t_{3} \approx t_{4}$ (or permutations thereof) where $t_{1} \approx t_{2}$ means that $t_{1}-t_{2}$ is a microscopic time..$^{20,21}$ 
The similarity between the right-hand sides of Eqs. (6) and (11) suggests that a relation exists between $\langle J\rangle_{E}$ and $\langle J(0), J(t)\rangle_{E}$. As a matter of fact, it is easy to show that Eqs. (6) and (11) imply

$$
\frac{d}{d E}\langle J\rangle_{E}=\frac{\beta}{2} \int_{-\infty}^{\infty}\langle J(0), J(t)\rangle_{E} d t
$$

This result is not approximate but a special case of an exact theorem due to Peterson. ${ }^{24}$

Since higher than second-order cumulants are zero for a Gaussian system, the maximum-entropy ansatz implies that the response is exactly linear whenever the equilibrium $J$ fluctuations are Gaussian. This is analogous to what happens in equilibrium statistical mechanics. A magnetic system, for instance, has a field-independent susceptibility if the free energy is quadratic in the magnetization. Note that non-Gaussian fluctuations survive in the thermodynamic limit, the central limit theorem notwithstanding. A simple example of this in statistical mechanics is energy fluctuations in the canonical ensemble. If the energy fluctuations are strictly Gaussian, the average energy is linear in $\beta$ so the specific heat varies as $T^{-2}$. Whenever this is not the case, the energy fluctuations are non-Gaussian.

The maximum-entropy formalism has a long history of applications to the problem of estimating the density matrix for a nonequilibrium system. This leads to a general- ization of equilibrium statistical mechanics, which takes into account the limited amount of information available about the system. ${ }^{25-29}$ Some applications of the formalism characterize the nonequilibrium system by its energy dissipation rate. ${ }^{30,31}$ As shown above, when applied to the external-field-induced "nonequilibrium" in the $J(t)$ space, this idea leads to definite predictions of the nonlinear response in terms of equilibrium fluctuations. An equivalent of Eq. (5) was discussed briefly by Bochkov and Kuzovlev, ${ }^{13}$ building on earlier work by Stratonovich. ${ }^{10}$ Only the case $u(t)=$ const was considered and the discussion was limited to Markovian systems. The Stratonovich theory is based on the assumption that, for jumps between two minima, the barrier to overcome is placed halfway between the two minima (in the direction of $Q$ ).

The maximum-entropy property of the ansatz shows that it is the most reasonable, given only a knowledge of the equilibrium fluctuations. This does not guarantee that the ansatz is correct, however. To look into this question, we first discuss the consequences of a phenomenological Langevin equation for $Q$ and then an exact result.

Suppose $Q$ obeys a Langevin equation $\dot{Q}=C F(Q)+\xi(t)$ where $F(Q)=F_{0}(Q)+E$ is the generalized force and $\xi(t)$ is a Gaussian white-noise term. Then the path probability is given by ${ }^{32,33}$

$$
P[Q(t)]=N^{-1} \exp \left[-\beta /(4 C) \int_{-\infty}^{\infty}[\dot{Q}(t)-C F(Q(t))]^{2} d t\right),
$$

or

$$
P_{E}[Q(t)]=N^{-1} P_{0}[Q(t)] \exp \left[\frac{\beta}{2} E \int_{-\infty}^{\infty} J(t) d t\right) \exp \left(-\frac{\beta}{2} E C \int_{-\infty}^{\infty} F_{0}[Q(t)] d t\right)
$$

The transformation from $Q(t)$ to $\dot{Q}(t)$ is linear. Therefore, Eq. (14) implies that the maximum-entropy ansatz leads to correct results whenever the last term is unimportant. As an example, consider again a particle in one dimension in a symmetric periodic potential at low temperature. Because of the $P_{0}[Q(t)]$ factor, the important paths fluctuate for some time around a potential-energy minimum before suddenly "jumping" to a new minimum. For these paths, the last term of Eq. (14) is almost independent of the number of minima traversed and may be ignored for the calculation of $\langle\Delta Q(t)\rangle_{E}$ for large $t$. Thus, the maximum-entropy ansatz leads to correct results in this case, as already shown in Eq. (9).

A result due to Furukawa sheds more light on the maximum-entropy ansatz. He showed that, for slowly fluctuating $J(t)$, the exact microscopic equation of motion implies ${ }^{34}$

$P_{E}[J(t)]=N^{-1} P_{0}[J(t)] \exp \left[\frac{\beta}{2} E \int_{-\infty}^{\infty} J(t) d t+O\left(E^{2}\right)\right]$.
Equation (15) suggests that there are two different ways a system may become nonlinear: either (i) as an interplay between non-Gaussian equilibrium $J$ fluctuations and the first-order term, or (ii) as a result of the higher-order terms becoming important. The first case is when the maximum-entropy ansatz gives correct results; this may be referred to as a "simple" nonlinearity since it results from the very same term in Eq. (15) that gives rise to the linear response. Upon increasing the external field, a system may pass from linear through simple nonlinear response to finally the "complex" nonlinear response due to the higher-order terms, or it may go directly from linear to complex nonlinear response. The maximumentropy ansatz is never correct for the latter systems, and in the former case it should only be trusted at not-toolarge external fields.

To summarize, it has been shown that the maximumentropy formalism in conjunction with statistical mechanics leads to unique predictions for the externalfield cumulant averages of $J(t)$ in terms of equilibrium averages. The ansatz Eq. (3) may be justified in other ways. It corresponds to keeping only the first-order term 
in Eq. (15) which is exact for slowly fluctuating $J(t)$. This term is responsible for the linear response, so the ansatz is clearly the simplest way to extrapolate linearresponse theory into the nonlinear regime. The extrapolation has the property that the response is exactly linear if the equilibrium $J$ fluctuations are Gaussian. If $Q$ obeys a Langevin equation, the ansatz is correct whenever the last term of Eq. (14) is unimportant; for slowly fluctuating $J(t)$, the requirement of consistency between Eqs. (14) and (15) implies that the extra terms of both equations must be insignificant and the maximum-entropy ansatz must be correct.

The maximum-entropy ansatz solves a conceptual problem, but open questions remain regarding the validity and the practical value of the ansatz. One obvious problem is that the ansatz predicts a universal form of the nonlinear response while one expects the response to depend on the way heat generated by the external field is removed. However, because the temperature enters into Eq. (5), the ansatz only makes sense in situations where the temperature is almost constant throughout the sample. In many cases, this requirement may be satisfied for a given external field by working with a sufficiently small sample; in this limit the problem of heating often becomes insignificant.

Equation (15) implies that the ansatz may be applied only at not-too-large external fields. Also, Eq. (15) implies that a system may become nonlinear either by first exhibiting the simple nonlinearity predicted by the ansatz before becoming complex nonlinear, or by going directly from the linear to the complex nonlinear regime. In this connection it should be noted that all formulas given above may be generalized to several degrees of freedom. One may speculate that, by including the "modecoupling" terms to other relevant degrees of freedom (e.g., the energy density), a complex nonlinearity may be converted to a simple nonlinearity, thus extending the validity of the ansatz. Even when the maximum-entropy ansatz is valid, the practical value of it is not clear at present. It is possible that the calculation of the equilibrium higher-order cumulant averages requires at least as much effort as a direct calculation of the nonlinear response. Certainly, this is the case for the previously discussed simple random walk in one dimension. On the other hand, Eq. (12) has provided insight into the problem of $1 / f$ noise in conductors. And, for instance, Eq. (10) gives explicit predictions for the response of a single atom to an external force, based on a knowledge of the equilibrium intermediate incoherent scattering function, a quantity which has been studied extensively. ${ }^{15}$

The author is indebted to Peter Wolynes for numerous helpful discussions and suggestions relating to this work, and also for his most kind hospitality during the time the work was carried out. This work was supported by the Danish Natural Science Research Council.
*Present and permanent address: IMFUFA, Roskilde Universitetscenter, P.O. Box 260, DK-4000 Roskilde, Denmark.

${ }^{1}$ H. Nyquist, Phys. Rev. 32, 110 (1928).

${ }^{2}$ L. Onsager, Phys. Rev. 37, 405 (1931); 38, 2265 (1931).

${ }^{3}$ J. G. Kirkwood, J. Chem. Phys. 14, 180 (1946).

${ }^{4}$ H. B. Callen and T. A. Welton, Phys. Rev. 83, 34 (1951).

${ }^{5}$ M. S. Green, J. Chem. Phys. 20, 1281 (1952).

${ }^{6}$ R. Kubo, J. Phys. Soc. Jpn. 12, 570 (1957).

${ }^{7}$ L. P. Kadanoff and P. C. Martin, Ann. Phys. (N.Y.) 24, 419 (1963).

${ }^{8}$ W. Bernard and H. B. Callen, Rev. Mod. Phys. 31, 1017 (1959).

${ }^{9}$ R. L. Stratonovich, Zh. Eksp. Teor. Fiz. 58, 1612 (1970) [Sov. Phys._JETP 31, 864 (1970)].

${ }^{10}$ R. L. Stratonovich, Vestn. Mosk. Univ. Fiz. Astronomiya 5, 16 (1962).

${ }^{11}$ E. T. Jaynes, Phys. Rev. 106, 620 (1957); 108, 171 (1957).

${ }^{12}$ The Maximum Entropy Formalism, edited by R. Levine and M. Tribus (MIT, Cambridge, MA, 1979).

${ }^{13}$ G. N. Bochkov and Yu. E. Kuzovlev, Zh. Eksp. Teor. Fiz. 72, 238 (1977) [Sov. Phys. - JETP 45, 125 (1977)].

${ }^{14}$ G. N. Bochkov and Yu. E. Kuzovlev, Physica 106A, 443 (1981); 106A, 480 (1981).

15J. P. Boon and S. Yip, Molecular Hydrodynamics (McGrawHill, New York, 1980).
${ }^{16}$ K. S. Singwi and A. Sjölander, Phys. Rev. 119, 863 (1960).

${ }^{17}$ D. A. Bell, J. Phys. C 13, 4425 (1980).

${ }^{18}$ P. Dutta and P. M. Horn, Rev. Mod. Phys. 53, 497 (1981).

${ }^{19}$ M. B. Weissman, Rev. Mod. Phys. 60, 537 (1988).

${ }^{20}$ C. J. Stanton and M. Nelkin, J. Stat. Phys. 37, 1 (1984).

${ }^{21}$ Th. M. Nieuwenhuizen and M. H. Ernst, J. Stat. Phys. 41, 773 (1985).

22J. Machta, M. Nelkin, Th. M. Nieuwenhuizen, and M. H. Ernst, Phys. Rev. B 31, 7636 (1985).

${ }^{23}$ J. C. Dyre, Phys. Rev. B 37, 10143 (1988).

${ }^{24}$ R. L. Peterson, Rev. Mod. Phys. 39, 69 (1967).

${ }^{25}$ E. T. Jaynes, in Statistical Physics Vol. 3 of 1963 Brandeis Lectures, edited by W. K. Ford (Benjamin, New York, 1963), p. 181.

${ }^{26}$ H. Schwegler, Z. Naturforsch. 20A, 1543 (1965).

${ }^{27}$ B. Robertson, Phys. Rev. 144, 151 (1966).

${ }^{28}$ D. N. Zubarev and V. P. Kalashnikov, Physica 46, 550 (1970).

${ }^{29}$ W. T. Grandy, Jr., Phys. Rep. 62, 175 (1980).

${ }^{30}$ H. Sato, Prog. Theor. Phys. 57, 1115 (1977).

${ }^{31}$ T. M. Brown, J. Phys. A 15, 2285 (1982).

${ }^{32}$ L. Onsager and S. Machlup, Phys. Rev. 91, 1505 (1953).

${ }^{33}$ H. Risken, The Fokker-Planck Equation (Springer, Berlin, 1984).

${ }^{34}$ H. Furukawa, Prog. Theor. Phys. 54, 370 (1975). 